\title{
A Class-based Probabilistic approach to Structural Disambiguation
}

\author{
Stephen Clark and David Weir \\ School of Cognitive and Computing Sciences \\ University of Sussex \\ Brighton, ]3N1. 9HQ, UK \\ \{stephecl, davidw\}@ocogs.susx.ac.uk
}

\begin{abstract}
Knowledge of which words are able to fill particular argument slots of a predicate can be used for structural disambiguation. This paper describes a proposal for acquiring such knowledge, and in line with much of the recent work in this area, a probabilistic approach is taken. We develop a novel way of using a semantic hicrarchy to estimate the probabilities, and demonstrate the gencral approach using a prepositional phrase attachment experiment.
\end{abstract}

\section{Introduction}

Knowledge of which words are able to fill particular argument slots of a predicate can be used for structural disambiguation. In the following example (Charniak, 1993), the fact that dog, rather than prize, is often the subject of run, can be used to decide on the attachment site of the rolative clause:

l'red awarded a prize for the dog that ran the fastest

We describe a proposal for acquiring such knowledge, and as in other recent work in this area (Resnik, 1993; Li and Abe, 1998), a probabilistic approach is taken. Using probabilities accords with the intuition that there are no absolute constraints on the arguments of predicates, but rather that constraints are satisfied to a certain degrec (Resnik, 1993). Unfortunately, defining probabilities in terms of words leads to a model with a vast number of parameters, resulting in a sparse data problem. 'Jo overcome this, we propose to define a probalility model in terms of senses from a semantic lierarchy, exploiting the fact that senses of nouns can be grouped together into semantically similar classes.

We use the semantic hierarchy of noun senses in WordNet (Fellbaum, 1998), which consists of 'lexicalised concepts' related by the 'is-a-kind- of 'relation. If $c$ ' is a kind of $c$, then $c$ is a $h y$ pernym of $c^{\prime}$, and $c^{\prime}$ a hyponym of $c$. Counts ase passed up the hierarchy from the senses of nouns appearing in the data. Thus if eat chicken appears in the data, the count for this item passes

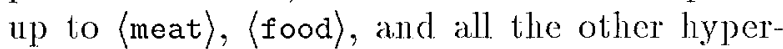
nyms of that sense of chicken. ${ }^{1}$ In order to estimate the probability that a scnse of chicken appears as the object of the verb eat, we represent (chicken) using a suitable hypernym, such as (food), and base our probability estimate on that instead. The level at which 〈chicken〉 is represented is crucial: it should be high enough for adequate counts to have accumulated, but not too high so that the hypernym is no longer representative of 〈chicken〉. An example of a hypernym which would be too high is 〈entity), as not all entities are semantically similar with respect to the object position of eat.

The problem of choosing an appropriate level in the lierarchy at which to represent a particular noun sense (given a predicate and argument position) has been investigated by Resnik (1993), Ii and Abe (1998) and Ribas (1995). The learning mechanism presented here is a novel approach based on finding semantically similar sets of concepts in a hierarchy. We demonstrate the effectiveness of our approach using a PP-attachment experiment.

\section{The Input Data and Semantic Hierarchy}

The data used to estimate the probabilities is a multiset of 'co-occurrence triples': a noun

\footnotetext{
${ }^{1}$ We use italics when referring to words, and angled brackets for concepts. This notation does not always pick ont a concept uniquely, but the context should make clcar the concept being referred to.
} 
lemma, verb lemma, and argument position.2 Let the universe of verbs, argument positions and nowns that can appear in the input data be denoted $\mathcal{V}=\left\{v_{1}, \ldots, v_{k_{v}}\right\}, R=$ $\left\{r_{1}, \ldots, r_{k_{\mathcal{K}}}\right\}$ and $\mathcal{N}=\left\{n_{1}, \ldots, n_{k_{\mathcal{N}}}\right\}$, respectively. Such data can be obtained from a tacebank, or from a shallow parser. Note that wo do not distinguish betweon alternative senses of verlos, and assume that each instance of a noun in the data relers to exactly one concept.

The scmantic hierarchy used is the noun hypernym taxomomy of WordNet (version 1.6).3 Let $\mathcal{C}=\left\{c_{1}, \ldots, c_{c}\right\}$ be the set of concepts in WordNet $\left(k_{c} \approx 60,000\right)$. $\Lambda$ concept is repre sented in Word Net by a syllset: a set of synonymons words which can be nsed to denote that concept. for example, the concept 'cocaine', as in the drug, is represented by the following syuset: \{cocainc, cocain, coke, snow, $\left.C^{\prime}\right\}$. let synl $(c) \subseteq \mathcal{N}$ be the synset tor the concept $c$, and let $\operatorname{cn}(n)=\{c \mid n \in \operatorname{syn}(c)\}$ be the set of concepts that can be denoted by the noun $n$.

The hicrarchy has the stucture of a directed acyclic graph, althongh the number of nodes in the graph with more than one parent is only around one percent of the total. The exlges in the graph form what we call the direct-isa relation (direct-isa $\subseteq C \times \mathcal{C}$ ). let isa $=\operatorname{dicect-isa{}^{*}}$ be the transitive, reflexive closure of diect-isa, so that $\left(c^{\prime}, c\right) \in \mathrm{isa} \Rightarrow c$ is a lyy] $)$ crnym of $c^{\prime}$; and let $\bar{c}=\left\{c^{\prime} \mid\left(c^{\prime}, c\right) \in\right.$ isa $\}$ be the set consisting of the concept $c$ and all of its liyponyms. 'l'huts, the set $\overline{\langle\text { food }\rangle}$ contains all the concepts which are kinds of food, including 〈food).

Note that words in the data can appear in synsets anywhere in the hierardy. Jven concopts such as 〈entity), which appear near the root of the hierarchy, have synsets containing words which may appeas in the data. The synset for 〈entity is $\{$ entily, something\}, and the words entity and something can appoar in the argument positions of verbs in the data.

\section{Probability Estimation}

'The problem being addressed in this section is to estimate $p(c \mid v, r)$, for $c \in \mathcal{C}, v \in \mathcal{V}$, and

\footnotetext{
${ }^{2}$ Only verbs are considered here, but this work applics to other predicates which take arguments that can be organised into a semantic hicrarchy.

${ }^{3}$ When we refer to concepts in WordNet, we mean concepts in WordNet's noun taxonomy.
}

$r \in \mathcal{R}$. The probability $p(c \mid v, r)$ is the probability that some noun in syn(c), when denoting concept $c$, appears in position $r$ of verb $v$ (given $r$ and $v$ ). Using the relative clause cxample from the introduction, the probabilitics $p(\langle\mathrm{dog}\rangle \mid r u n$, subj $)$ and $p(\langle$ prize $\rangle \mid r u n$, subj $)$ can be compared to decide on the attachment site in Fred awarded a prize for the dog that ran the fastest. We expect $p(\langle$ dog $\rangle \mid r u n$, subj $)$ to bo groater than $p(\langle$ prize $) \mid$ run, subj). Although tho focus is on $p(c \mid v, r)$, the techniques described here can be used to estimate other probabilitics, such as $p(c, r \mid v)$. (In fact, the latter probability is used in the P'P-attachment experinents described in Section 5.)

Using maximum likelihood to estimate $p(c \mid v, r)$ is not viable because of the huge number of parancters involved. Many combinations of $c, v$ and $r$ will not oceur in the data. 'lo reduce the number of paraneters which need to be estimated, we utilise the fact that concepts can be grouped into classes, and represent $c$ using a class $c^{\prime}$, for some hypernym $c^{\prime}$ of $c$. Howcver, $p\left(\overline{c^{\prime}} \mid v, r\right)$ cannot be lsed as an estimate of $p(c \mid v, r)$, as $p(\bar{c} \mid v, r)$ is given by the following:

$$
p\left(c^{\prime} \mid v, r\right)=\sum_{a^{\prime \prime} \in \overline{c^{\prime}}} p\left(c^{\prime \prime} \mid v, r\right)
$$

The probability $p\left(\overline{c^{\prime}} \mid v, r\right)$ increases as $c^{\prime}$ moves up the hicrarchy. lot example, $p(\overline{\langle\text { food }\rangle} \mid$ eal,olj $)$ is not a good estimate of $p(\langle$ chicken $\rangle \mid e a t$, olj $)$. What can be done though, is to condition on sets of concepts, and use the probability $p\left(v \mid \overline{c^{\prime}}, r\right)$. If it can be shown that $p\left(v \mid \overrightarrow{c^{\prime}}, r\right)$, for some hypernym $c^{\prime}$ of $c$, is a reasomable cstimate of $p(v \mid c, r)$, then we have a way of estimating $p(c \mid v, r)$. To get $p(v \mid c, r)$ from $p(c \mid v, r)$ Bayes rule is used:

$$
p(c \mid v, r)=p(v \mid c, r) \frac{p(c \mid r)}{p(v \mid r)}
$$

'The probabilities $p(c \mid r)$ and $p(v \mid r)$ can be estimated using maximum likelihood estimates, as the conditioning cvent is likely to occur often cnough for sparse data not to be a problem. (Alternatively one could back-off to $p(c)$ and $p(v)$ respectively, or use a lincar combination of $p(c \mid r)$ and $p(c)$, and $p(v \mid r)$ and $p(v)$, respectively.) 'The formulae for these estimates will be given shortly. 'This only leaves $p(v \mid c, r)$. The 
proposal is to estimate $p($ cat $\mid\langle$ chicken $\rangle$, obj) using $p(\operatorname{cat} \mid \overline{\langle\text { food }\rangle}$, obj $)$, or something similar. The following proposition shows that if $p\left(v \mid c^{\prime \prime}, r\right)$ is the same for each $c^{\prime \prime}$ in $\overline{c^{\prime}}$, where $c^{\prime}$ is some hypernym of $c$, then $p\left(v \mid \overrightarrow{c^{\prime}}, r\right)$ will be equal to $p(v \mid c, r)$ :

$$
p\left(v \mid c^{\prime \prime}, r\right)=k \text { for all } c^{\prime \prime} \in \overline{c^{\prime}} \Rightarrow p\left(v \mid \overline{c^{\prime}}, r\right)=k
$$

The proof is as follows:

$$
\begin{aligned}
p\left(v \mid \overline{c^{\prime}}, r\right) & =p\left(\overline{c^{\prime}} \mid v, r\right) \frac{p(v \mid r)}{p\left(\overline{c^{\prime}} \mid r\right)} \\
& =\frac{p(v \mid r)}{p\left(\overline{c^{\prime}} \mid r\right)} \sum_{c^{\prime \prime} \in \overline{c^{\prime}}} p\left(c^{\prime \prime} \mid v, r\right) \\
& =\frac{p(v \mid r)}{p\left(\overline{c^{\prime}} \mid r\right)} \sum_{c^{\prime \prime} \in \overline{c^{\prime}}} p\left(v \mid c^{\prime \prime}, r\right) \frac{p\left(c^{\prime \prime} \mid r\right)}{p(v \mid r)} \\
& =\frac{1}{p\left(\overline{c^{\prime}} \mid r\right)} k \sum_{c^{\prime \prime} \in \overline{c^{\prime}}} p\left(c^{\prime \prime} \mid r\right) \\
& =k
\end{aligned}
$$

So in order to estimate $p(v \mid c, r)$, we need a way of searching for a set $c^{\prime}$, where $c^{\prime}$ is a hypernym of $c$, which consists of concepts $c^{\prime \prime}$ which have similar $p\left(v \mid c^{\prime \prime}, r\right)$. Of course we cannot $c x-$ pect to find a set consisting of concepts which have identical $p\left(v \mid c^{\prime \prime}, r\right)$, which the proposition strictly requires, but if the $p\left(v \mid c^{\prime \prime}, r\right)$ are similar, then we can expect $p\left(v \mid \overline{c^{\prime}}, r\right)$ to be a reasonable estimate of $p(v \mid c, r)$. We refer to the set $\overline{c^{\prime}}$ as the 'similarity-class' of $c$, and the suitable hypernym, $c^{\prime}$, as top $(c, v, r)$. The next section $\mathrm{cx}-$ plains how we determine similarity classes. The maximum likelihood estimates for the relevant probabilities are given in Table $1 .^{4}$

\section{Finding Similarity-classes}

First we explain how we determine if a set of concepts has similar $p\left(v \mid c^{\prime \prime}, r\right)$ for each concept $c^{\prime \prime}$ in the set. Then we explain how we determine top $(c, v, r)$.

\footnotetext{
${ }^{4}$ Since we are assuming the data is not sense disambiguated, freq $(c, v, r)$ cannot be obtaincd by simply counting senses. The standard approach, which is adopted here, is to estimate freq $(c, v, r)$ by distributing the count for each noun $n$ in syn $(c)$ evenly among all senses of the noun. Yarowsky (1992) and Resnik (1993) explain how the noise introduced by this technique tends to dissipate as counts are passed up the hicrarcly.
}

Table 1: Maximum Likelihood Estimates freq $(c, v, r)$ is the number of $(n, v, r)$ triples in the data in which $n$ is being used to denote $c$.

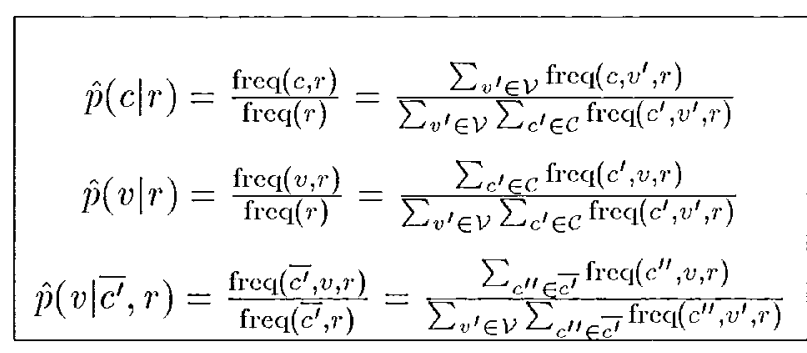

The method used for comparing the $p\left(v \mid c^{\prime \prime}, r\right)$ for $c^{\prime \prime}$ in some set $\overline{c^{\prime}}$, is based on the technique in Clark and Weir (1999) used for finding homogeneous sets of concepts in the WordNet noun hierarchy. Rather than directly compare estimates of $p\left(v \mid c^{\prime \prime}, r\right)$, which are likely to be unreliable, we consider the children of $c^{\prime}$, and use estimates based on counts which have accumulated at the children. If $c^{\prime}$ has children $c_{1}^{\prime}, c_{2}^{\prime}, \ldots, c_{n}^{\prime}$, we compare $p\left(v \mid \overline{c_{i}^{\prime}}, r\right)$ for each $i$. This is an approximation, but if the $p\left(v \mid \overline{c_{i}^{\prime}}, r\right)$ are similar, then we assume that the $p\left(v \mid c^{\prime \prime}, r\right)$ for $c^{\prime \prime}$ in $\bar{c}^{\prime}$ are similar too.

To determine whether the children of some hypernym $c^{\prime}$ have similar $p\left(v \mid \overrightarrow{c_{i}^{\prime}}\right)$, where $c_{i}^{\prime}$ is the $i$ th child, we apply a $\chi^{2}$ test to a contingency table of frequency counts. Table 2 shows some example frequencies for $c^{\prime}$ equal to 〈nutriment>, in the object position of eat. The figures in. brackets are the expected values, based on the marginal totals in the table. The null hypothcsis of the test is that $p\left(v \mid \overline{c_{i}^{\prime}}, r\right)$ is the same for each $i$. For Table 2 the null hypothesis is that for every child, $c_{i}^{\prime}$, of (nutriment $\rangle$, the probability $p\left(c a t \mid \overline{c_{i}^{\prime}}\right.$,obj $)$ is the same.

The log-likelihood $\chi^{2}$ statistic corresponding to T'able 2 is 4.8 . The $\log$-likelihood $\chi^{2}$ statistic is used rather than the Pcarson's $\chi^{2}$ statistic because it is thought to be more appropriate when the counts in the contingency table are low (Dunning, 1993). This tends to occur when the test is being applied to a set of concepts near the foot of the hierarchy. ${ }^{5}$ We compared

\footnotetext{
${ }^{5}$ Fisher's exact test conld be used for tables with low counts, but we do not do so because tables dominated by low counts are likely to have a ligh percentage of noise, due to the way counts for a noun are split among
} 
Table 2: Contingency table for children of (nutriment

\begin{tabular}{|c|c|c|c|c|c|}
\hline$\overline{c_{i}}$ & \multicolumn{2}{|c|}{$\hat{\operatorname{freq}}\left(\overline{c_{i}}, c a l, o b \mathrm{j}\right)$} & \multicolumn{2}{|c|}{$\begin{array}{l}\hat{\operatorname{req}}\left(\overline{c_{i}}, o b \mathrm{j}\right)- \\
\hat{\operatorname{req}}\left(\overline{c_{i}}, c a l, o b j\right)\end{array}$} & $\begin{array}{l}\hat{\operatorname{rrc}}\left(\overline{c_{i}}, \text { obj }\right)= \\
\sum_{v \in \mathcal{V}} \operatorname{frcal}\left(\overline{c_{i}}, v, \mathrm{obj}_{j}\right)\end{array}$ \\
\hline$\overline{\langle\mathrm{milk}\rangle}$ & 0.0 & $(0.6)$ & 9.0 & $(8.4)$ & 9.0 \\
\hline$\overline{\langle\text { meal }\rangle}$ & 8.5 & $(5.6)$ & 78.0 & $(80.9)$ & 86.5 \\
\hline$\langle$ course & 1.3 & $(1.7)$ & 24.7 & $(24.3)$ & 26.0 \\
\hline$\overline{\langle\text { dish }\rangle}$ & 5.3 & $(5.7)$ & 82.3 & $(81.9)$ & 87.6 \\
\hline \multirow[t]{2}{*}{$\langle$ delicacy $\rangle$} & 0.3 & $(1.8)$ & 27.4 & $(25.9)$ & 27.7 \\
\hline & \multicolumn{2}{|c|}{15.4} & \multicolumn{2}{|c|}{221.4} & 236.8 \\
\hline
\end{tabular}

the performance of log-likelitood $\chi^{2}$ and Pearson's $\chi^{2}$ using the P'P-attaclument experiment described in Section 5. It was found that the log-likelihood $\chi^{2}$ test did perform slightly better. Jior a significance level of 0.05 (whijch is the level used in the experiments), with 1 degrees of frecdom, the critical value is 14.86 (llowell, 1997). Thus in this case, the null hypothesis would not be rejected.

In order to determine top $(c, v, r)$, we compare $p\left(v \mid \overline{c_{i}}, r\right)$ for the children of the hypernyms of c. Initially top $(c, v, r)$ is assigned to be the concept $c$ itself. Then, by worling up the hierauchy. top $(c, v, r)$ is reassigned to be successive hypernyms of $c$ until the siblings of top $(c, v, r)$ have significantly different probabilities. In cases where a concept has more than one parent, the parent is chosen which results in the lowest $x^{2}$ value as this indicates the $p\left(v \mid \overline{c_{i}}, r\right)$ are more similar. The set $\overline{t o p}(c, v, r)$ is the similarityclass of $c$ for verb $v$ and position $r$.

The next section provides cvidence that the technique for choosing top $(c, v, r)$, which we call the 'similarity-class' technique, does select an appropriate level of generalisation.

\section{Experiments using PP-attachment ambiguity}

The Pl'attachment problem we address considers 4-tuples of the form $n, n_{1}, p r, n_{2}$, and the problem is to decide whether the prejositional phrase pr $n_{2}$ attaches to the verb $v$ or the noun $n_{1}$. For example, in the following case the problem is to decide whether

alternative senses. We rely on the log-likclihood $x^{2}$ test returning a non-significant result in these cases. from minisler attaches to awail or approval: await approval from minister

We chose the p'l'attaclument problem because P'P-attachment is a pervasive form of ambiguity, and there exist standard training and test data, which makes for easy comparisons with other approaches. This problem has been tackled by a number of researchers. Brill and Resnik (1994), Ratnaparkihi et al. (1994), Collins (1995), '/atvrel and Daelemans (1997) all report results between $81 \%$ and $85 \%$, with Stetina and Nagao (1997) reporting a result of $88 \%$, which matches the human performance on this task reported by Ratnaparkhi et al. (1994).

Although the P'Pattachnent, problem has characteristics that make it suitable for evaluation, it presents a much bigger sparse data problem than would be expected in other problems such as relative clause attachment. 'J'lie reason for this is that we need to consider how a concept is associated with combinations of predicates and prepositions. The appproach described here uses probabilities of the form $p(c, p \mid v)$ and $p\left(c, p r \mid n_{1}\right)$, where $c \in \operatorname{cn}\left(n_{2}\right)$. T'his means that for many predicate/preposition combinations which occur infrequently in the data, there are few examples of $n_{2}$ which can be used for populating WorlNet in these cases. Despite this, we were still able to carry out an evaluation by considering sulbsets of the test data for which the relevant predicate/preposition combinations did occur frequently in the training datia.

We decide on the attachment site by compar- 
ing $p\left(c_{v}, p r \mid v\right)$ and $p\left(c_{n_{1}}, p r \mid n_{1}\right)$, where

$$
\begin{aligned}
c_{v} & =\arg \max _{c \in \operatorname{cn}\left(n_{2}\right)} p(c, p r \mid v) \\
c_{n_{1}} & =\arg \max _{c \in \operatorname{con}\left(n_{2}\right)} p\left(c, p r \mid n_{1}\right)
\end{aligned}
$$

The sense of $n_{2}$ is chosen which maximises the relevant probability in each potential attachment case. If $p\left(c_{v}, p r \mid v\right)$ is grcatcr than $p\left(c_{n_{1}}, p r \mid n_{1}\right)$, the attachment is made to $v$, otherwise to $n_{1}$. If $n_{2}$ is not in WordNet we compare $p(p r \mid v)$ and $p\left(p r \mid n_{1}\right)$. Probabilities of the form $p(c, p r \mid v)$ and $p\left(c, p r \mid n_{1}\right)$ are used rather than $p(c \mid v, p r)$ and $p\left(c \mid n_{1}, p r\right)$, because the association between the preposition and $v$ and $n_{1}$ contains useful information. In fact, for a lot of cases this information alone can be used to decide on the correct attachment site. The original corpus-based method of Hindle and Rooth (1993) used exactly this information. Thus the method described here can be thought of as Ilindle and Rooth's method with additional classbased information about $n_{2}$.

In order to estimate $p\left(c_{v}, p r \mid v\right)$ (and $\left.p\left(c_{n_{1}}, p r \mid n_{1}\right)\right)$ we apply the same procedure as described in Section 3, first rewriting the probability using Bayes' rule:

$$
\begin{aligned}
p\left(c_{v}, p r \mid v\right) & =p\left(v \mid c_{v}, p r\right) \frac{p\left(c_{v}, p r\right)}{p(v)} \\
& =p\left(v \mid c_{v}, p r\right) \frac{p\left(p r \mid c_{v}\right) p\left(c_{v}\right)}{p(v)}
\end{aligned}
$$

The probabilities $p\left(c_{v}\right)$ and $p(v)$ can be estimated using maximum likclihood estimates, and $p\left(v \mid c_{v}, p r\right)$ and $p\left(p r \mid c_{v}\right)$ can be estimated using maximum likelihood estimates of $p\left(v \longdiv { \operatorname { t o p } ( c _ { v } , v , p r ) }, p r\right)$ and $p\left(p r \mid \overline{\operatorname{top}\left(c_{v}, p r\right)}\right)$ respectively. ${ }^{6}$

We used the training and test data described in Ratnaparklii et al. (1994), which was taken from the Penn 'Treebank and has now become the standard data set for this task. The data set consists of tuples of the form $\left(v, n_{1}, p r, n_{2}\right)$, together with the attachment site for each tuple. There is also a development set to prevent implicit training on the test sct during development. We extracted $\left(v, p r, n_{2}\right)$ and $\left(n_{1}, p r, n_{2}\right)$

\footnotetext{
${ }^{6}$ In Section 4 we only gave the procedure for determining top $\left(c_{v}, v, p r\right)$, but top $\left(c_{v}, p r\right)$ can be determined in an analogous fashion.
}

triples from the training set, and in order to increase the number of training triples, we also extracted triples from unambiguous cases of at tachment in the Penn Treebank. We preprocessed the training and test data by lemmatising the words, replacing numerical amounts with the words 'definite_quantity', replacing monetary amounts with the words 'sum_of_money' etc. We then ignored those triples in the resulting training set (but not test set) for which $n_{2}$ was not in WordNet, which left a total of 66,881 triples of training data. The test set contains 3,097 examples.

Table 3 gives some examples of the extent to which the similarity-class technique is gencralising, using the training data just described, and a significance level of 0.05 . The chosen hypernym is shown in upper casc. Note that the WordNet hicrarchy consists of nine separate sub-hicrarchies, headed by such concepts as 〈entity), 〈abstraction〉, 〈psychological feature〉, but we assume the existence of a single root which dominates cach of the sub-hierarchies, which is referred to as $\langle$ root $\rangle$. In cases where WordNet is very sparsely populated, it is prefcrable to go to 〈root), rather than stay at the root of one of the subhierarchies where the data may be noisy or too sparse to be of any use. The table shows that with the amount of data available from the Treebank, the similarity-class technique is selecting a level at or close to 〈root〉 in many cases.

We compared the similarity-class technique with fixing the level of generalisation. 'Two fixed levels were used: the root of the entire liierarchy ( $\langle$ root $\rangle$ ), and the set consisting of the roots of each of the 9 sub-hicrarchies. The procedure which always sclects $\langle$ root $\rangle$ ignores any information about $n_{2}$, and is equivalent to comparing $p(p r \mid v)$ and $p\left(p r \mid n_{1}\right)$, which is the Hindle and Rooth approach. The results on the 3,097 test cases are shown in Table 4. We used a significance level $\alpha$ of 0.05 for the $\chi^{2}$ test. ${ }^{7}$

As the table shows, the disambiguation accuracy is below the state of the art. However, the results are comparable with those of $\mathrm{I} i$ and

\footnotetext{
${ }^{7}$ Similar results were obtained using alternative levels of significance. Rather than simply selecting a value for $\alpha$ such as $0.05, \alpha$ can be treated as a parameter of the model, whose optimum value can be obtained by running the disambiguation method on some held-ont sujervised data.
} 
Iable 3: Ilow the similarity-class technique chooses $\operatorname{top}(c, v, p r)$ and top $\left(c, n_{1}, p r\right)$

\begin{tabular}{|c|c|}
\hline$\left(n_{1}, p r, c\right)$ & Iypernyms of $c$ \\
\hline $\begin{array}{c}(\text { bid, for },\langle\text { company }\rangle) \\
(\text { concern, about },\langle\text { risk }\rangle) \\
(\text { billion, in, }\langle\text { cash }\rangle)\end{array}$ & $\begin{array}{l}\langle\text { company }\rangle\langle\text { establishment }\rangle \text { (organisation }\rangle\langle\text { social_group }\rangle\langle\text { GROUP }\rangle\langle\text { root }\rangle \\
\langle\text { risk }\rangle\langle\text { venture }\rangle\langle\text { task }\rangle\langle\text { work }\rangle\langle\text { activity }\rangle\langle\text { act }\rangle\langle\text { ROOT }\rangle \\
\langle\text { cash }\rangle\langle\text { currency }\rangle\langle\text { monetary_system }\rangle\langle\text { asset }\rangle\langle\text { POSSESSION }\rangle\langle\text { root }\rangle\end{array}$ \\
\hline$(v, p r, c)$ & \\
\hline $\begin{array}{c}(\text { notify,of, }, \text { transaction }\rangle) \\
(\text { close, } a t,\langle\text { definite-quantity }\rangle) \\
(\text { mect, with, } \text { official }\rangle)\end{array}$ & $\begin{array}{l}\langle\text { transaction }\rangle\langle\text { group_action }\rangle\langle\text { act }\rangle\langle\text { ROOT }\rangle \\
\langle\text { DEFINITE_QUANTITY }\rangle\langle\text { measure }\rangle\langle\text { abstraction }\rangle\langle\text { root }\rangle \\
\langle\text { official }\rangle\langle\text { adjudicator }\rangle\langle\text { person }\rangle\langle\text { Iife_form }\rangle\langle\text { CAUSAL_AGENT }\rangle\langle\text { entity }\rangle\langle\text { root }\rangle\end{array}$ \\
\hline
\end{tabular}

Table 4: Complete test set 3097 test cases

\begin{tabular}{|l|l|}
\hline Gencralisation technique & $\%$ correct \\
\hline Sinilarity-class & 80.3 \\
Select root of sub-hierarchy & 77.9 \\
Always select $\langle$ root & 79.0 \\
\hline
\end{tabular}

Abe (1998) who adopt a similar approach using Word Net, but with a different training and test set. li and $\Lambda$ be improved on the llin. dle and Rooth technicue by $1.5 \%$, which is in line with our results. As an evaluation of the similarity-class technigue, the result is inconclusive. The reason for this is that when the technique was being used to estimate $p\left(v \mid c_{v}, p r\right)$ and $p\left(n_{1} \mid c_{n_{1}}, p r\right)$, in many cases the root of the hierarchy was being chosen as the appropriate level of generalisation, due to a sparsely populated WordNet in that instance. Recall that this is largely due to the fact that we are attempting to populate WordNet for combinations of predicates and prepositions. In such cases the similarity-class technique is not helping because there is very little or no information about $n_{2}{ }^{8}$

\footnotetext{
${ }^{8}$ In an effort to ohtain more clata we applied the extraction henristic of Ratnaparkhi (1998) to Wall Strect Journal text, which increased the number of training triples by a factor of 10. This only achieved comparable results, however, presumably because the ligh volume: of noise in the data ontweights the benefit of the increase in data size. Ratnaparkhi reports only $69 \%$ accuracy for
}

Table 5: 〈root〉 being selected for both attachment points - 113 test cases

\begin{tabular}{|l|l|}
\hline Generalisation technique & $\%$ correct \\
\hline Similarity-class & 90.3 \\
Solect root of sub-hicharchy & 81.4 \\
Always select $\langle$ root & 79.6 \\
\hline
\end{tabular}

Thable 6: 〈root〉 being selected for at most one of the attachment points 10;32 test cases

\begin{tabular}{|l|l|}
\hline Gencralisation technigue & \% correct \\
\hline Similarity-class & 88.1 \\
Select root of sub-hiemarelyy & 85.5 \\
Always select (root) & 85.5 \\
\hline
\end{tabular}

In order to evaluate the similarity-class technicue further, we took those test cases for which the root was not being selected when estimating both $p\left(v \mid c_{v}, m r\right)$ and $p\left(n_{1} \mid c_{n_{1}}, p r\right)$. This applied to 113 cases. The results are given in Table 5. We also took those test cases for which the root was being selected when estimating at most one of $p\left(v \mid c_{\eta}, p r\right)$ and $p\left(n_{1} \mid c_{n_{1}}, p r\right)$. 'l'his appliced to 1032 test cases. The results are shown in Thab]e 6 .

the extraction lieuristic when applied to the P'om Trecbank (excluding cases where the preposition is of). 


\section{Conclusions}

We have shown that when instances of WordNet are well populated with examples of $n_{2}$, the method described, here for solving PP-attachment ambiguities is highly accurate. When WordNet is sparsely populated, the method automatically resorts to comparing just the preposition and each of the potcntial attachment sites, as the similarity-class technique will select $\langle$ root $\rangle$ as the appropriate level of generalisation for $n_{2}$ in such cases. We have also shown the similarity-class technique to be superior to using a fixed level of generalisation in WordNet.

Further work will look at how to integrate probabilities such as $p(c \mid v, r)$ into a model of dependency structure, similar to that of Collins (1996) and Collins (1997), which can be used for parse selection. However, knowledge of selectional preferences cannot by itself solve the problem of structural disambiguation, and this further work will also look at using additional knowledge, such as subcatcgorisation information.

\section{References}

Lric Brill and Philip Resnik. 1994. A rulc-based approach to prepositional phrase attachment disambiguation. In lroceedings of the fifleenth International Conference on Compulational Linguistics.

Eugcne Charniak. 1993. Statistical Language Learning. 'The MI'T' l'ress.

Stephen Clark and David Weir. 1999. An iterative approach to estimating frequencics over a semantic hierarchy. In Proceedings of the Joint SIGDAT' Conference on Empirical Methods in Natural Language Processing and Very Large Corpora, pages 258-265.

Michael Collins. 1995. Prepositional phrase attachment through a backed-off model. In Proccedings of the Third Workshop on Very Large Corpora, pages 27-38, Cambridge, Massachusetts.

Michael Collins. 1996. A new statistical parser based on bigram lexical dependencics. In Procecdings of the 3/th Annual Meeting of the ACI, pages 184-191.

Michacl Collins. 1997. Three generative, lexicalised models for statistical parsing. In Proceedings of the 35th Annual Meeting of the
Association for Compulational Linguistics, pages $16-23$.

Ted Dunning. 1993. Accurate methods for the statistics of surprise and coincidence. Compulational Linguistics, 19(1):61-74.

Christiane Fellbaum, editor. 1998. WordNet An Electronic Lexical Database. The MIT Press.

Donald Hindle and Mats Rooth. 1993. Structural ambiguity and lexical relations. Computational Linguistics, 19(1):103-120.

1)avid Howell. 1997. Statistical Methods for l'sychology: 4th ed. Duxbury Press.

IIang Li and Naoki Abe. 1998. Generalizing case frames using a thosaurus and the MDI, principle. Compulational Linguistics, $24(2): 217-214$.

Adwait Ratnaparklii, Jeff Reynar, and Salim Roukos. 1994. A maximum entropy model for prepositional phrase attachment. In Proceedings of the ARPA IIuman Language Technology Workshop, pages 250-255.

Adwait Ratnaparkhi. 1998. Unsupervised statistical models for prepositional phrase attachment. In Proceedings of the Seventeenth International Conference on Computational Iinguistics, Montreal, Canada, Aug.

Plilip Resnik. 1993. Selection and Information: A Class-Based Approach to Lexical Rolationships. P'l.IJ. thesis, University of Pennsylvania.

Francesc Ribas. 1995. On learning more appropriate selectional restrictions. In Proceedings of the Seventh Conference of the liuropean Chapter of the Association for Computational linguistics, I) ublin, Ircland.

Jiri Stetina and Makoto Nagao. 1997. Corpus based P'P attachment ambiguity resolution with a scmantic dictionary. In Procecdings of the Fifth Workshop on Very Large Corpora, pages 66-80, Bcijing and Hong Kong.

David Yarowsky. 1992. Word-sense disambiguation using statistical models of Roget's categories trained on large corpora. In Proceedings of COLING-92, pages 454-460.

Jakub Zavrel and Walter Daelemans. 1997. Memory-based learning: Using similarity for smoothing. In Proceedings of $A C l / E A C L$ 97 , Madrid, Spain. 